Research Paper

\title{
Knockdown of Long Non-coding RNA HOTAIR Suppresses Tumor Invasion and Reverses Epithelial- mesenchymal Transition in Gastric Cancer
}

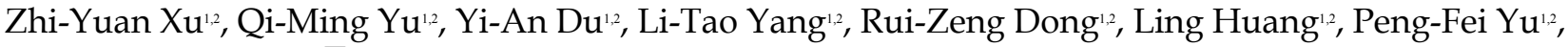 \\ Xiang-Dong Cheng ${ }^{1,2,}, \mathbb{D}$ \\ 1. Department of Abdominal Surgery, Zhejiang Cancer Hospital, Hangzhou, Zhejiang, China; \\ 2. Zhejiang Cancer Research Institute, Zhejiang Cancer Center, Hangzhou, Zhejiang, China. \\ $\triangle$ Corresponding author: Prof. Xiangdong Cheng, Department of Abdominal Surgery, Zhejiang Cancer Hospital, GuangJi Road 38\#, \\ Hangzhou, 310022, Zhejiang, China. Tel: +86-571-88122011. Fax: +86-571-88122011; E-mail: abcsurg@gmail.com \\ (c) Ivyspring International Publisher. This is an open-access article distributed under the terms of the Creative Commons License (http://creativecommons.org/ \\ licenses/by-nc-nd/3.0/). Reproduction is permitted for personal, noncommercial use, provided that the article is in whole, unmodified, and properly cited.
}

Received: 2013.03.27; Accepted: 2013.06.12; Published: 2013.06.28

\begin{abstract}
Background: Over-expression of long non-coding RNA HOTAIR has been reported in several types of cancer. Yet its involvement in gastric cancer (GC) has not been well understood. The aim of present study was to examine the expression pattern of HOTAIR in GC patients, then, explore its role in promoting cancer invasion and underlying molecular mechanism. Methods: The expression level of HOTAIR in the tumor specimens of GC patients was quantified by Realtime RT-PCR. The correlation between HOTAIR level and clinicopathological factors as well as prognosis was then examined. Down-regulation of HOTAIR by RNA interference was applied to investigate its roles in tumor invasiveness via the view of Epithelial-to-mesenchymal transition (EMT). Results: The expression level of HOTAIR in cancer tissues was higher than that in adjacent noncancerous tissues. Expression level of HOTAIR was significantly correlated with lymph node metastasis and TNM stage. Furthermore, high expression level of HOTAIR was a predictor of poor over-all survival in GC patients. In vitro, inhibition of HOTAIR in GC cells could reduce invasiveness, as well as the expression of MMPI and MMP3. In addition, suppression of HOTAIR could reverse EMT process. Conclusions: HOTAIR could act as a potential predictor for over-all survival in patients with GC. Inhibition of HOTAIR could reduce invasiveness and reverse EMT process in GC cells, indicating the potential role of HOTAIR in GC diagnostics and therapeutics.
\end{abstract}

Key words: Epithelial-mesenchymal Transition, Gastric cancer, HOTAIR, Long non-coding RNA, Lymphatic node metastasis.

\section{Introduction}

Gastric cancer (GC) is a major health problem and the second highest cause of cancer-related mortality worldwide [1], approximately $60 \%$ of GC cases are diagnosed in Asia [2] and China has the most cases, accounting for $42 \%$ of all GC worldwide [3]. Despite the improvement in surgical techniques and adjuvant therapy, this cancer is still highly lethal because of its aggressive metastasis and the fact that it is often diagnosed at an advanced stage. There is need to improve an understanding of disease causing mechanisms and the identification of specific biomarkers for GC progression may aid in both prediction and improvement of clinical outcome.

By using molecular techniques, several genes that are differentially activated in GC have been identified. Major attention of these efforts has focused 
on characterization of expression of protein-coding genes. However, the majority of the human genome consists of non-protein-coding RNA (ncRNA), representing $>98 \%$ of the total genome sequence [4]. According to their size, ncRNAs are subdivided into two groups: small ncRNAs (< $200 \mathrm{nt})$ and long ncRNAs(lncRNA)[5, 6]. Small ncRNAs including well-documented microRNAs receive the most attention and are shown to play many important roles in cancer [7]. In contrast, lncRNAs were initially argued to be spurious transcriptional noise. However, increasing discoveries of misregulated lncRNA expression across numerous cancer types have suggested that aberrant lncRNA expression may be a major contributor to tumorigenesis [8,9]. Consequently, if we want to fully understand the complex mechanisms underlying malignant processes, e.g., carcinogenesis, metastasis, and drug resistance, we need to consider the role of lncRNAs [10].

The recent finding that lncRNA HOTAIR can promote cancer metastasis furthers the relevance of lncRNAs to human cancer. The overexpression of HOTAIR directs Polycomb Repressive Complex 2 (PRC2) to silence HOXD9, a locus involved in developmental pattern [11]. A subsequent study reveals that HOTAIR is over-expressed in approximately one quarter of human breast cancers, targeting PRC2 to approximately 800 ectopic sites in the genome, which leads to changes in gene expression [12]. Recently, Over-expression of HOTAIR has also been documented in several other types of human cancer including Hepatocellular cancer (HCC) [13] and pancreatic cancer [14], suggesting an oncogenic function for this lncRNA. Yet, its potential involvement in GC is so far unknown.

In the present study, therefore, we explored HOTAIR expression pattern and its correlation with clinicopathological factors in GC patients. Then, its prognostic significance was assessed. The effect of HOTAIR on cell invasiveness was investigated in vitro. Furthermore, the molecular mechanism of HOTAIR activity in tumor metastasis was also addressed in this study.

\section{Materials and Methods}

\section{Patients and Samples}

83 cases of patients who underwent D2 radical resection between March 2006 and June 2010 were recruited from our institute. The study was approved by the Ethics Committee of our hospital. Specimens were obtained immediately after surgical resection and stored at $-80^{\circ} \mathrm{C}$ for further analysis. Lymph nodes (LNs) with or without metastatic were also harvested during gastrectomy. There were 56 men and 27 women, ranging in age from 27 to 81 years, with a median age of 55 years. Tumor stage was defined according to American Joint Committee on Cancer/International Union Against Cancer tumor, node, metastasis (TNM) classification system (the seventh edition). Clinical data such as date of birth, sex, date of surgery, serum CEA level, HP status, tumor size, location of tumor and other content of histopathological reports were extracted from computerized clinical database.

\section{Cell Lines}

All the cell lines were purchased from American Type Culture Collection (Manassas, VA) or Shanghai Institute of Cell Biology (Shanghai, China) and preserved in our institute. All of the cell lines were maintained in the recommended culture conditions and incubated at $37^{\circ} \mathrm{C}$ in a humidified environment containing $5 \% \mathrm{CO} 2$.

\section{Plasmids and Transfection}

The siRNAs sequences targeting human HOTAIR (siRNA-1 UAACAAGACCAGAGAGC UGUU; siRNA-2 CCACAUGAACGCCCAGAGAUU; siRNA-3 GAACGGGAGUACAGAGAGAUU) or negative control RNA (NC CUACAACAGCC ACAACGUCdTdT) were designed according to previous study [15] and produced by Genepharma (Shanghai, China). Full length human snail and twist gene were amplified and cloned into a pcDNA3.1(-) vector (Invitrogen, CA) as described previously[16]. SiRNA and Plasmids were transfected into cells with lipofectamine 2000 (Invitrogen, CA).

\section{Total RNA Extraction, Reverse Transcription and realtime $R T-P C R$}

Total RNA from tissues and cells was extracted using Trizol reagent (Invitrogen, CA). Reverse transcription for mRNAs was performed using the M-MLV Reverse Transcriptase (TaKaRa, Dalian, China). The cDNA template was amplified by real-time RT-PCR using the SYBR® Premix Dimmer Eraser kit (TaKaRa, Dalian, China). GAPDH was used as an internal control, and HOTAIR values were normalized to GAPDH. Real-time RT-PCR reactions were performed by the ABI7500 system (Applied Biosystems, CA). The relative expression fold change of mRNAs was calculated by the $2^{-\Delta \Delta C t}$ method.

\section{Western Blot Analysis}

Proteins were extracted with RIPA (Beyotime, Shanghai, China), separated by $8 \%-12 \%$ NUPAGE bis-tris Gel (Invitrogen, CA) and transferred onto polyvinylidene difluoride membranes. The following process was finished as decribed[17]. The primary 
antibodies were listed in Supplementary Material: Table S1.

\section{Invasion assay}

Cell-invasion assay was performed using a transwell assay (Millipore, Billerica, MA). The details of this method had been described [16]. The number of cells placed in the upper chamber was $3 \times 10^{4}$. The stained cells were counted under an inverted microscope (5 fields per membrane). Each experiment was performed in triplicate.

\section{Immunofluorescence}

Cell $\left(5 \times 10^{5}\right)$ were implanted onto a cell culture dish (NEST Biotech, Hong Kong) for 24 hours. Cells were fixed with paraformaldehyde for 30 minutes, then permeabilized with $0.1 \%$ Triton X-100 for $5 \mathrm{~min}$ at room temperature, the primary antibodies was added after permeabilization, After incubation in the dark for 24 hours at $4^{\circ} \mathrm{C}$, cells were incubated with alexa flours $488 \mathrm{TgG}$ donkey anti-mouse or anti-rabbit (1:500, Invitrogen, USA) for an hours at room temperature, Nuclei were stained with propidium iodide(PI) for 5 minutes when necessary. Fluorescence images were photographed with a confocal microscopy (Leica DMIRE2, Germany)

\section{A}

\section{Relative HOTAIR}

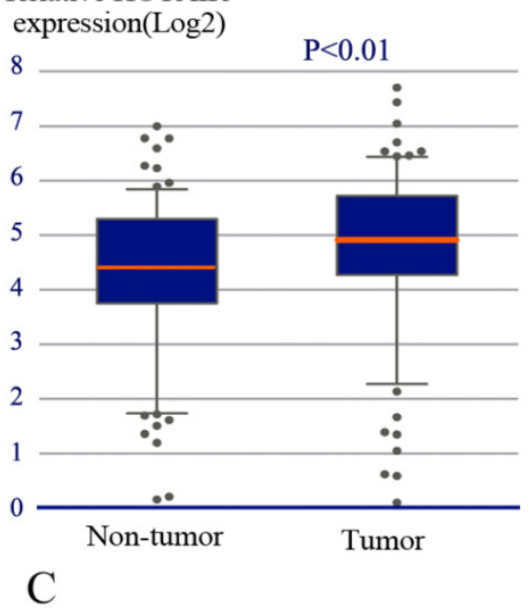

Relative HOTAIR expression $(\log 2)$

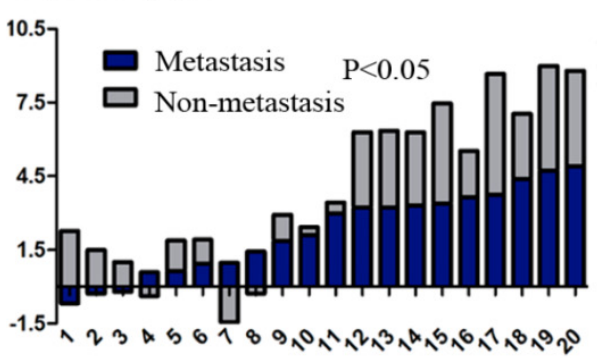

B
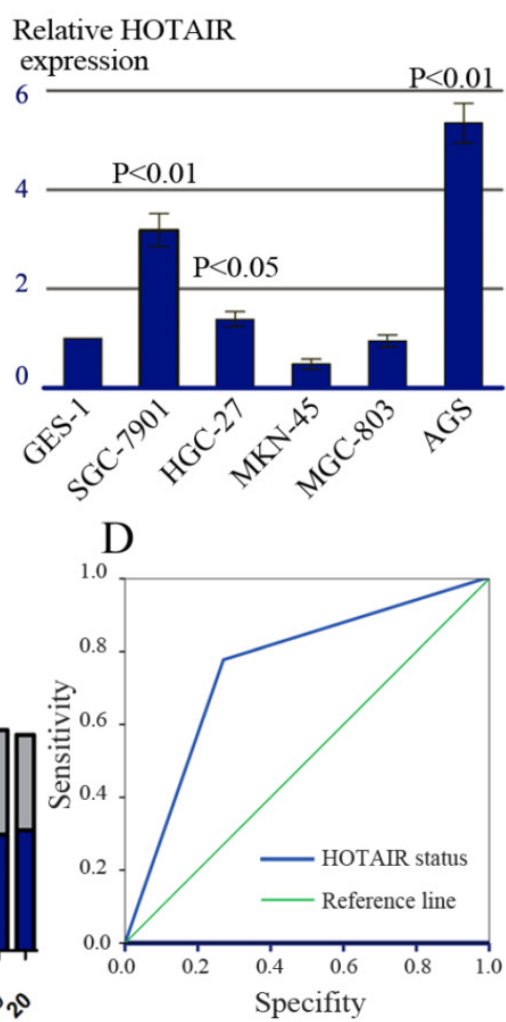

\section{Statistical Analysis}

Comparisons of continuous data were analyzed by the independent $t$ test or paired $t$ test between the 2 groups, whereas categorical data were analyzed by the chi-square test. Over-all survival was analyzed by the Kaplan-Meier method, and the differences between groups were estimated by the log-rank test. Independent prognostic indicators were assessed in the multivariate analysis using Cox's proportional hazard model. All statistical analyses were performed using SPSS for Windows v.16.0 (SPSS, Chicago, IL) and GraphPad Prism 5.0 (GraphPad Software, La Jolla, CA). $\mathrm{P}<0.05$ was considered statistically significant.

\section{Results}

\section{Over-expression of HOTAIR in Human GC}

Realtime RT-PCR was performed to evaluate the expression of HOTAIR in 83 pairs of human GC and adjacent noncancerous tissues. Over-expression of HOTAIR was detected in 56/83 $(67.0 \%)$ of GCs as compared to their non-tumorous counterparts $(P=0.008$, Figure $1 \mathrm{~A})$, indicating that HOTAIR was frequently over-expressed in GC. Furthermore, the expression level of HOTAIR in 6 cell lines including one immortalized gastric cell lines (GES-1) and 5 GC cell lines (AGS,SGC-7901,MKN-45,HGC-27,MGC803) was determined by realtime RT-PCR. We observed that the expression of HOTAIR in AGS, SGC-7901and HGC-27 cells was higher than that in GES-1 cell (Figure 1B).

We next evaluated the relationship between HOTAIR expression and various clinicopathological parameters. High expression of HOTAIR was positively correlated with advanced TNM stage $(P=$ 0.013 ), lymphatic nodes (LNs) metastasis $(P=0.002)$. There was no significant correlation between HOTAIR expression and sex, age, location of tumor, size of tumor, liver metastasis, Lauren's classification or serum CEA levels (Table 1).

Fig I. HOTAIR expression and its clinical significance. (A) The relative expression of HOTAIR was measured by real-time RT-PCR in tumorous and adjacent non-tumorous tissues. (B) The relative expression of HOTAIR was measured in various cell lines, including one immortalized gastric cell lines (GES-I) and $5 \mathrm{GC}$ cell lines (AGS, SGC-790I, MKN-45, HGC-27, MGC-803). (C) The relative expression of HOTAIR mRNA in LNs with or without metastasis. (D) ROC analysis of HOTAIR expression in predicting the existence of LNs metastasis. 
Table I. The relationship between HOTAIR expression and clinicopathological feature of 83 GC patients.

\begin{tabular}{|c|c|c|c|c|c|}
\hline \multirow[t]{2}{*}{ Clinicopatholocical variables } & \multirow[t]{2}{*}{ Number of each group } & \multicolumn{2}{|c|}{ Hotair expression } & \multirow[t]{2}{*}{$\mathrm{X}^{2}$} & \multirow[t]{2}{*}{$P$ value } \\
\hline & & high & low & & \\
\hline All case & 83 & 56 & 27 & & \\
\hline \multicolumn{6}{|l|}{ Age(years) } \\
\hline$\leqq 50$ & 32 & 23 & 9 & 0.46 & 0.497 \\
\hline$>50$ & 51 & 33 & 18 & & \\
\hline \multicolumn{6}{|l|}{ gender } \\
\hline Male & 56 & 37 & 19 & 0.153 & 0.695 \\
\hline female & 27 & 19 & 8 & & \\
\hline \multicolumn{6}{|l|}{ HP } \\
\hline positive & 56 & 40 & 16 & 1.229 & 0.268 \\
\hline negative & 27 & 16 & 11 & & \\
\hline \multicolumn{6}{|l|}{ Size of tumor, $(\mathrm{cm})$} \\
\hline$<5($ small $)$ & 41 & 27 & 14 & 0.096 & 0.756 \\
\hline$\geqq 5$ (large) & 42 & 29 & 13 & & \\
\hline Location of tumor & & & & 0.24 & 0.887 \\
\hline Cardia & 17 & 12 & 5 & & \\
\hline Body & 20 & 14 & 6 & & \\
\hline antrum & 46 & 30 & 16 & & \\
\hline Depth of tumor invasion & & & & 3.14 & 0.37 \\
\hline $\mathrm{T} 1$ & 9 & 4 & 5 & & \\
\hline $\mathrm{T} 2$ & 13 & 9 & 4 & & \\
\hline $\mathrm{T} 3$ & 25 & 16 & 9 & & \\
\hline $\mathrm{T} 4$ & 26 & 27 & 9 & & \\
\hline Lymph node metastasis & & & & 9.576 & 0.002 \\
\hline present & 75 & 52 & 17 & & \\
\hline Absent & 8 & 4 & 10 & & \\
\hline Liver metastasis & & & & 0.104 & 0.747 \\
\hline Absent & 74 & 49 & 25 & & \\
\hline present & 9 & 7 & 2 & & \\
\hline Invasion of contiguous organs $\mathrm{n}(\%)$ & & & & 2.009 & 0.147 \\
\hline Yes & 15 & 13 & 2 & & \\
\hline No & 68 & 43 & 25 & & \\
\hline vessel invasion & & & & 2.126 & 0.145 \\
\hline Negative & 49 & 30 & 19 & & \\
\hline positive & 34 & 26 & 8 & & \\
\hline Stage & & & & 6.179 & 0.013 \\
\hline I, II & 25 & 12 & 13 & & \\
\hline III, IV & 58 & 44 & 14 & & \\
\hline Lauren's classification & & & & 2.808 & 0.094 \\
\hline diffuse & 22 & 18 & 4 & & \\
\hline intestinal & 61 & 38 & 23 & & \\
\hline Grade of differentiation & & & & 1.59 & 0.207 \\
\hline Well and moderate & 29 & 17 & 12 & & \\
\hline Poor and not & 55 & 39 & 15 & & \\
\hline Preoperative chemotherapy & & & & 0.654 & 0.419 \\
\hline Yes & 34 & 18 & 16 & & \\
\hline no & 49 & 38 & 11 & & \\
\hline Serum CEA value (ug/L) & & & & 2.987 & 0.084 \\
\hline$<5$ & 51 & 38 & 13 & & \\
\hline$\geqq 5$ & 32 & 18 & 14 & & \\
\hline Serum CA199 value(U/L) & & & & 0.974 & 0.324 \\
\hline$<37$ & 62 & 40 & 22 & & \\
\hline$\geqq 37$ & 21 & 16 & 5 & & \\
\hline
\end{tabular}




\section{HOTAIR Over-expression was Frequently Detected in LNs with Metastasis}

LNs metastasis is one of the most important prognostic factors in patients with GC. To further explore the role of HOTAIR in LNs metastasis, HOTAIR expression was compared between 20 paired of LNs specimens by using realtime RT-PCR. Each paired LNs specimen consisted of a LN with metastasis and a LN without metastasis derived from the same patient. In all, 14/20 pairs of LNs $(70.0 \%)$ showed higher expression of HOTAIR in metastatic LNs compared with their matched non-metastatic LNs $(P=0.035$, Figure $1 C)$.

In addition, we explored whether HOTAIR expression status in primary tumor could predict the existence of LNs metastasis. The predictive values were studied by ROC analysis. Our result showed the area under the curve was 0.755 (Figure 1D).

\section{Some EMT- and Metastasis-related Genes were Misregulated in GC with High Expression of HOTAIR}

EMT- and metastasis -related genes play an important role in promoting invasion. In order to iden-

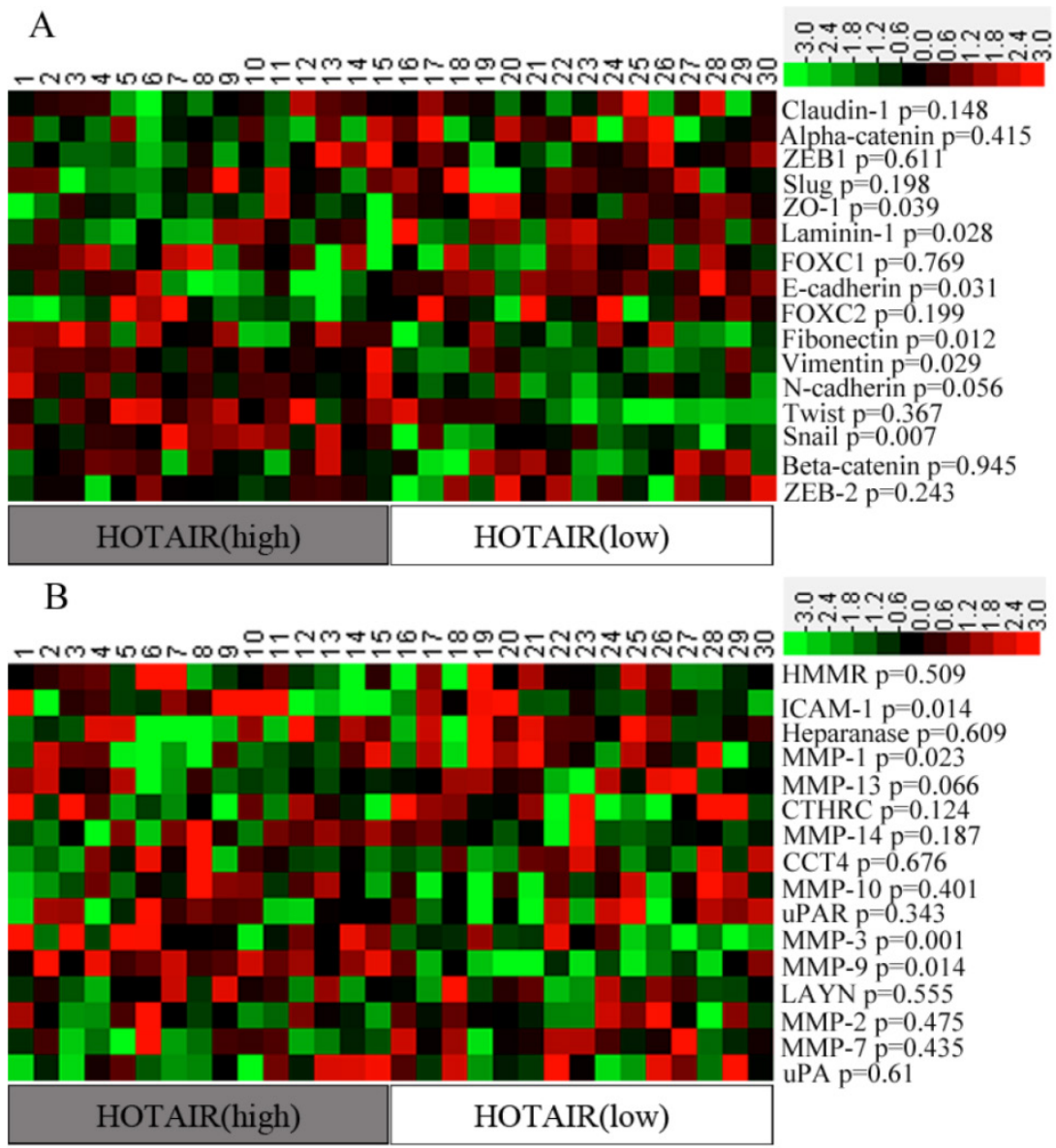

Fig 2. Expression level of EMT- and metastasis-related genes in two groups of patients. (A) The Heat-map representation of the EMT-related genes. (B) The Heat-map representation of the metastasis-related genes. Genes are plotted from the minimal level of expression (green) to the maximal level (red). tity a "metastasis signature" of genes related to HOTAIR expression, we first calculated the "expression ratio" of HOTAIR in tumorous to that in non-tumorous tissues (tumorous: non-tumorous). Then, all the samples were divided into two groups according to their expression ratios: the HOTAIR (High) group, representing the top 15 cases of the expression ratios, and the HOTAIR (Low) group, representing the lowest 15 cases of the expression ratios. The remaining cases were excluded from further analyses. Expression levels of some EMT- and metastasis-related genes were compared between 2 groups. Our results showed that most of the epithelial markers were down-regulated in HOTAIR (High) patients when compared with that in HOTAIR (Low) patients, Whereas, most of the mesenchymal markers were up-regulated in HOTAIR (High) group, although some difference was not statistically significant. A heat map also showed that 4 metastasis -related genes (e.g., ICAM-1, MMP1, MMP3, and MMP9) were significantly over-expressed in HOTAIR (High) group $(\mathrm{p}<0.05)$ (Figure 2). 


\section{Inhibition of HOTAIR Reduced Invasiveness of GC Cells}

To further examine the functional role of HOTAIR in GC cells, AGS cells, which expressed high level of endogenous HOTAIR, were transfected with siRNA duplexes against HOTAIR. The successful knockdown of HOTAIR expression was confirmed by real-time RT-PCR (Figure3A). Transwell Matrigel invasion assays showed that down-regulation of HOTAIR by siRNAs caused significant decrease in the cell invasiveness (Figure 3B).

We also performed western-blot to determine the alternation of some MMPs expression after HOTAIR deletion. As anticipated, HOTAIR silencing significantly suppressed the expression level of MMP1 and MMP3 (Figure 3C).

\section{HOTAIR Silencing Reversed EMT through Regulating Snail}

We next tested whether direct inhibition of HOTAIR could reverse EMT in GC cell line. Both AGS and SGC-7901 cell lines, which expressed high level of endogenous HOTAIR, were transfected with siRNA-3. The morphological changes of AGS cells after transfected with siRNA-3, including the acquirement of tight adhesion to each other and rounded morphology, suggested the reversal of EMT (Figure 4A). However, these typical changes were not easily observed in SGC-7901 cells, though some marginal cells turned into the phenotype more similar to epithelial cells after HOTAIR inhibition (data not shown). We then compared the expression of phenotypic markers and found that knockdown of HOTAIR led to decreased expression of some mesenchymal markers including vimentin, and $\mathrm{N}$-cadherin. Meanwhile, the expression of epithelial markers such as E-cadherin and ZO-1 were elicited in response to HOTAIR silencing, though the change of $\mathrm{ZO}-1$ were not consistent between 2 cell lines (Figure 4B). A series of transcription factors (TFs) are involved in various EMT processes, therefore, we assessed the effect of HOTAIR silencing on the expression of following TFs known to promote EMT: snail, slug, twist ,FOXC1and zeb1, we found HOTAIR-siRNA AGS cells expressed lower level of snail and twist compared with the control cells (Figure 4B). In addition, the expression of Snail was also decreased significantly, while the expression of Twist and Slug decreased slightly, the expression of ZEB1 and FOXC1 remained unchanged in SGC-7901 cells after HOTAIR knockdown (Figure $4 C)$.

Next, we sought to unravel whether HOTAIR promoted GC progression via activating Snail or Twist expression. AGS cells were co-transfected with HOTAIR siRNA-3, combined with pcDNA3.1(-)-snail or pcDNA3.1(-)-twist for evaluation of invasion ability. Realtime RT-PCR was used to confirm the validity of these co-transfections (Figure 5B). Results showed that GC invasiveness, suppressed by HOTAIRsiRNA, could be restored by exogenous snail, rather than twist (Figure 5A).

We further examined whether exogenous Snail expression could restore the EMT, which had been reversed by HOTAIR knockdown. AGS cells were transfected with negative control, HOTAIR-siRNA-3 alone or HOTAIR-siRNA-3 combined with pcDNA3.1(-)-snail. Our results showed that transient treatment with pcDNA3.1(-)-snail could restore the expression state of E-cadherin, vimentin and $\mathrm{N}$-cadherin, all of which were significantly regulated by HOTAIR knockdown (Figure 5C).
A

Relative HOTAIR

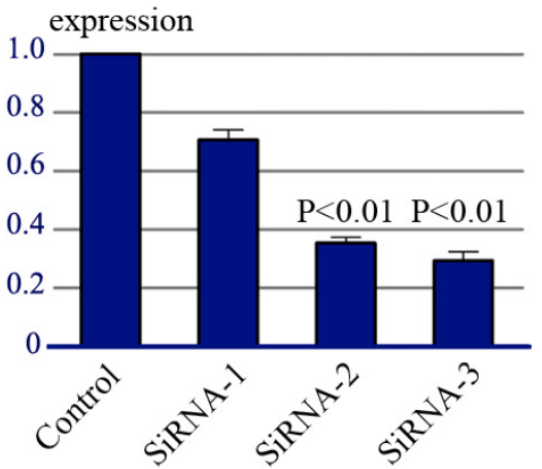

B

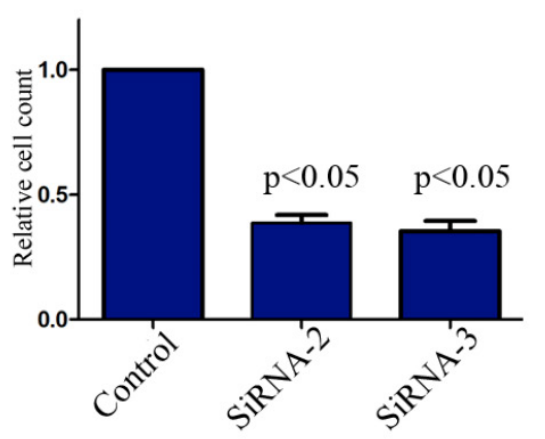

$\mathrm{C}$

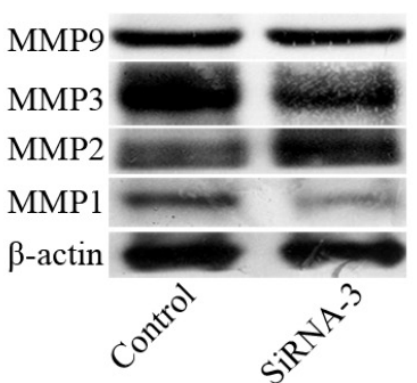

Fig 3. Effect of HOTAIR knockdown on the invasiveness. (A) Knockdown of HOTAIR expression in AGS cells by siRNAs was confirmed by real-time RT-PCR. (B) Transwell matrigel invasion assays of AGS cells after transfected with siRNA-2 and siRNA-3. (C) Western-blot tested the expression level of some MMPs in the AGS cells after HOTAIR knockdown. 


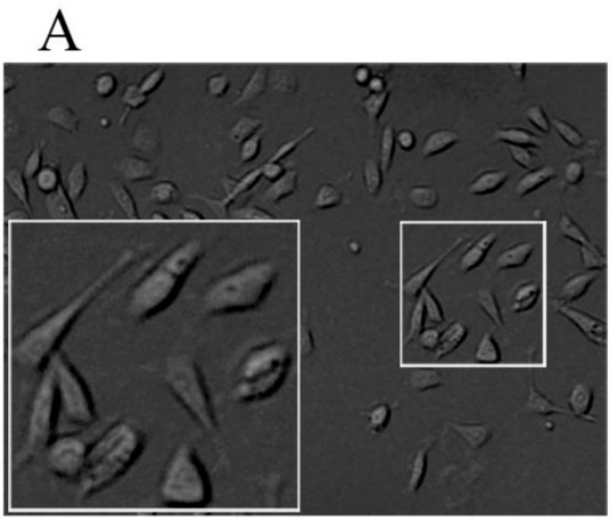

Control

B

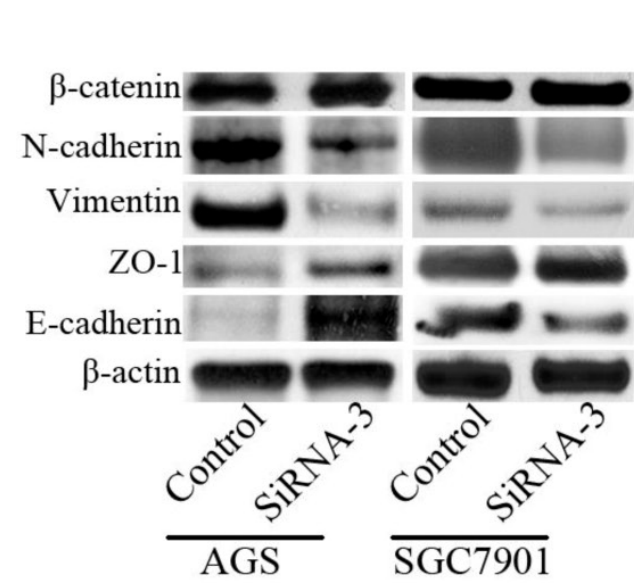

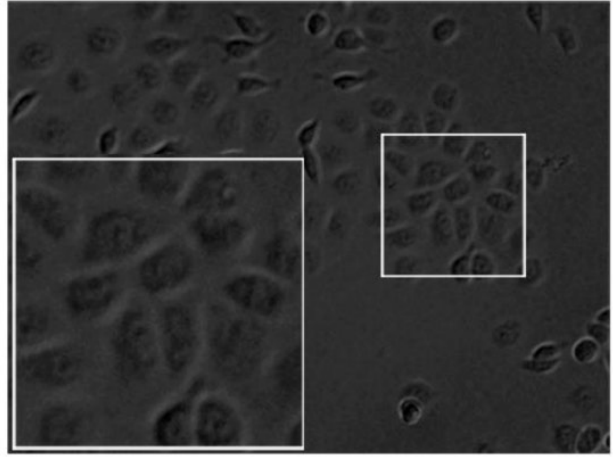

HOTAIR-siRNA-3

$\mathrm{C}$

FOXC1

ZEB1

Twist

Slug

Snail

$\beta$-actin
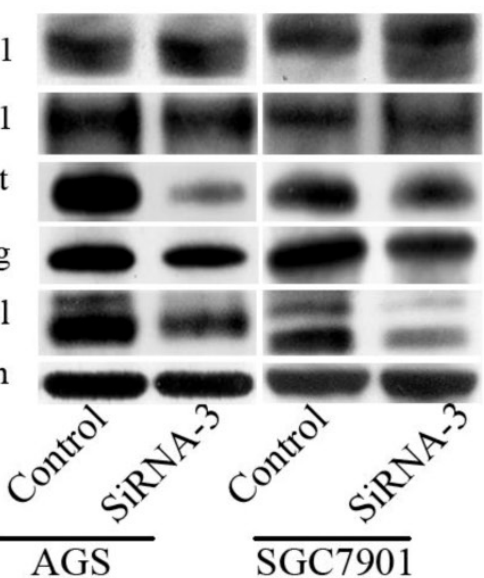

$\mathrm{D}$
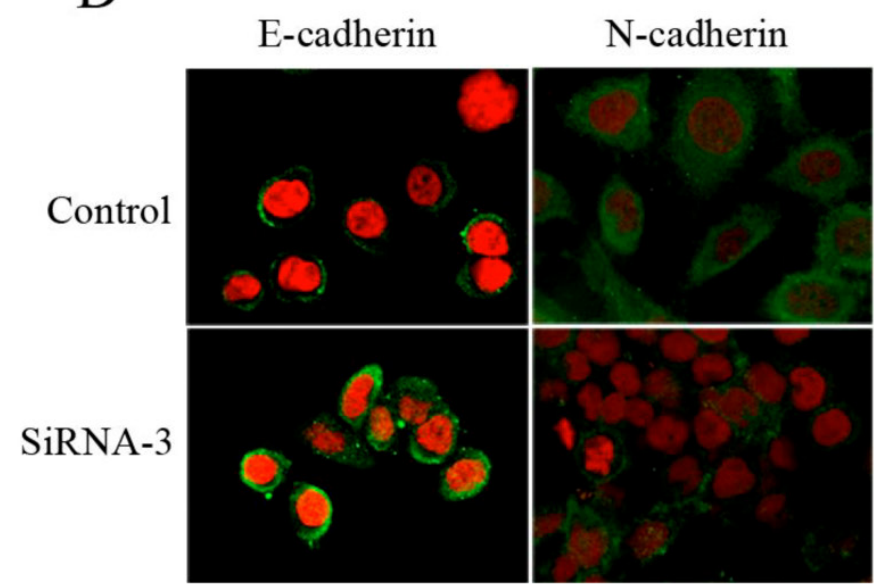

Vimentin

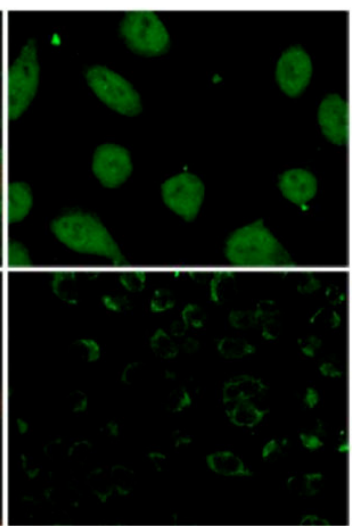

Fig 4. Effect of HOTAIR knockdown on the EMT progress. (A) Morphological change of AGS after transfected with HOTAIR-siRNA-3. (B) Western-blot analysis of phenotypic markers after HOTAIR knockdown in GC cells. (C) Western-blot analysis of EMT-related TFs after HOTAIR knockdown in GC cells. (D) Confocal microscopy analysis of phenotypic markers in AGS cells. The green signal represented the staining of corresponding proteins, and the red signal represented the nuclear DNA staining by PI. (Magnification: $\times 1200$ ). 


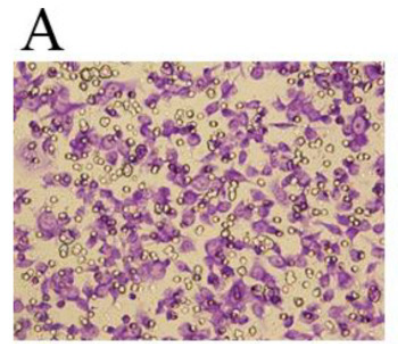

Control

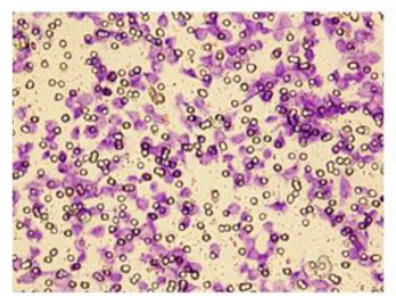

SiRNA-3+snail

B

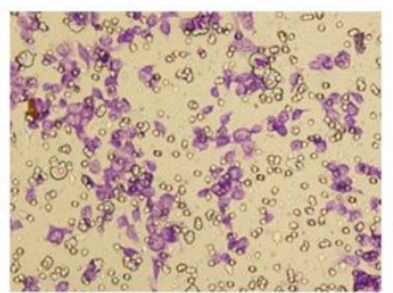

SiRNA-3

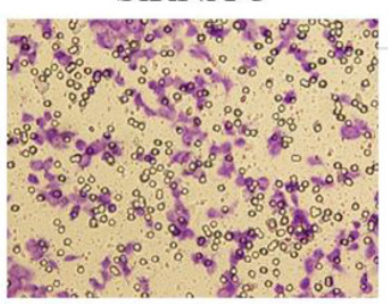

SiRNA-3+twist
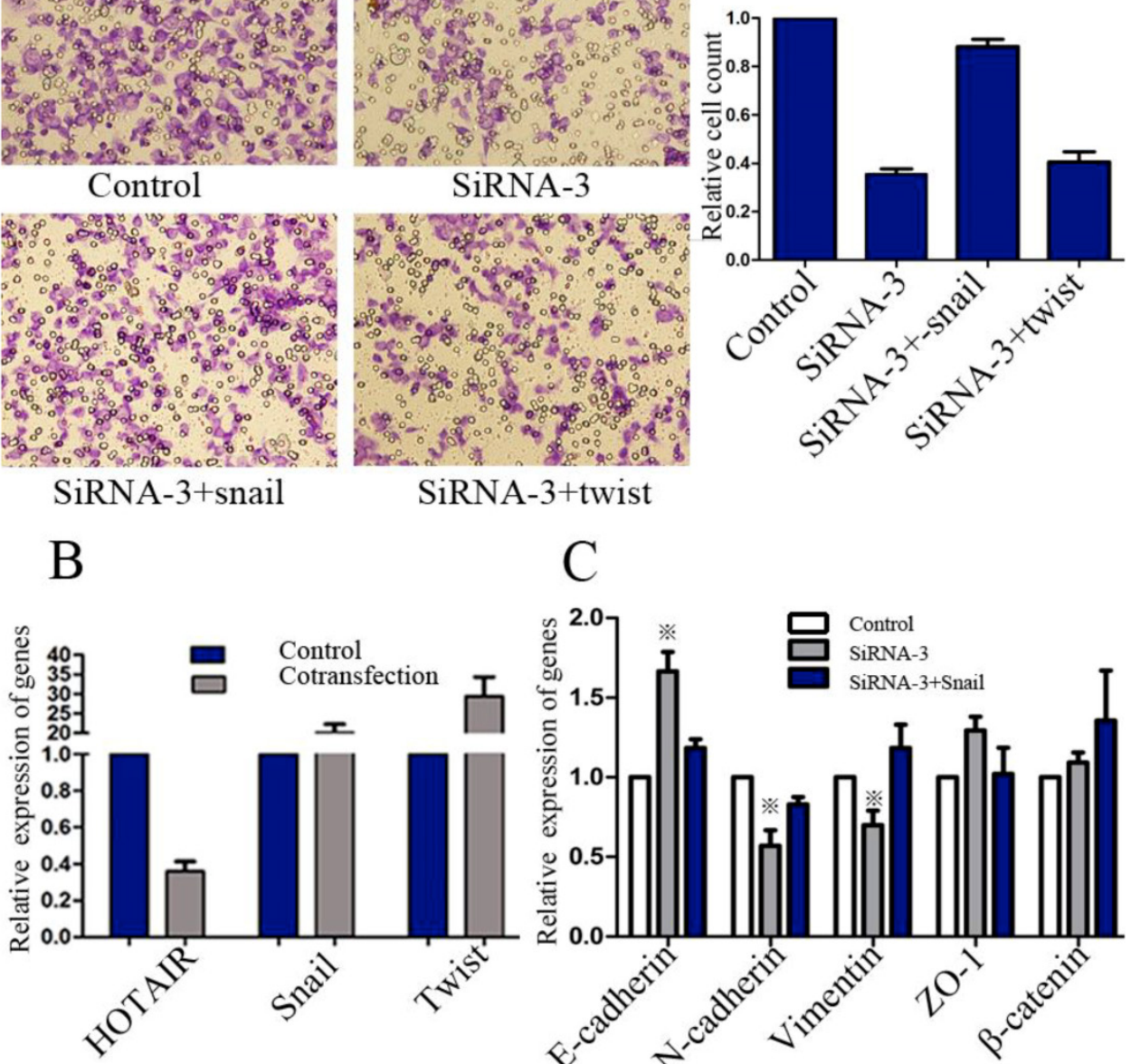

$\mathrm{C}$

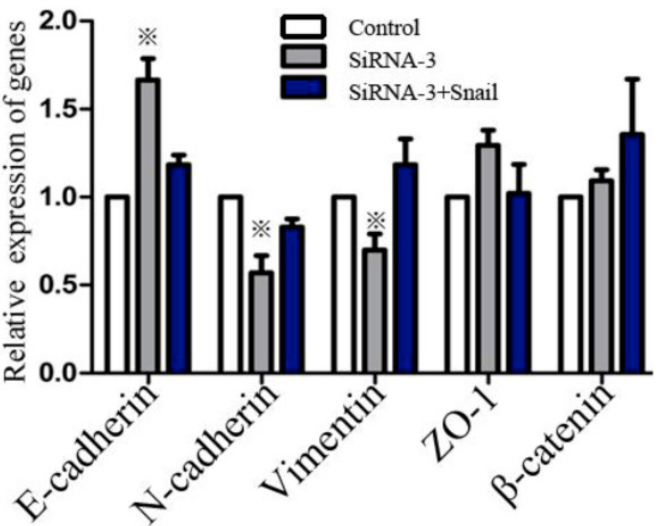

Fig 5. Effect of exogenous snail or twist on invasiveness and EMT of GC cells. (A) Transwell matrigel invasion assays of AGS cells after transfected with negative control, HOTAIR-siRNA-3 alone or HOTAIR-siRNA-3 combined with exogenous snail or twist. (B) The efficiency of transfections was confirmed by real-time RT-PCR. (C) Realtime RT-PCR analysis of phenotypic markers of AGS cells after transfected with negative control, HOTAIR-siRNA-3 alone or. HOTAIR-siRNA-3 combined with exogenous snail ( $※$ : the difference, compared with control group, was significant).

\section{HOTAIR and Clinical Outcome}

The 1-, 3- and 5-year cumulative survival rates were $81 \%, 66 \%$, and $57 \%$ respectively, for patients with low HOTAIR expression, and $78 \%, 50 \%$ and $31 \%$, respectively, for those with high HOTAIR expression. Clearly, GC patients with high expression of HOTAIR had a poorer prognosis than those with low HOTAIR expression (Figure 6A). Univariate analysis indicated that the factors significantly associated with survival were invasion depth, TNM stage, HOTAIR level, preoperative chemotherapy, LNs metastasis, and Lauren's classification. Whereas histological type, gender, age, serum CEA levels, and et al, were not related to the prognosis of the patients (Table 2). The clinicopathological parameters that were correlated with the survival of the patients in the univariate analysis were included in the multivariate Cox analysis. It was indicated that TNM stage, LNs metastasis, HOTAIR expression were independent prognostic factors for patients with GC (Table 2).

When stratified by Lymphatic node status, it was found that most of our patients located in N2 and N3a stage. In the both stages, the patients with low expression of HOTAIR had significantly longer mean survival times than those with high expression of HOTAIR. (Figure 6B, C). 
Table 2. Univariate and multivariate analyses of factors associated with overall survival.

\begin{tabular}{|c|c|c|c|c|}
\hline \multirow[t]{2}{*}{ Clinicopatholocical variables } & \multirow{2}{*}{$\begin{array}{l}\text { Univariate } \\
P\end{array}$} & \multicolumn{3}{|l|}{ multivariate } \\
\hline & & Hazard rate & $95 \% \mathrm{CI}$ & $P$ \\
\hline Age(years): $\leqq 50$ versus $>50$ & 0.101 & & & NA \\
\hline Gender: male versus female & 0.653 & & & NA \\
\hline HP: positive versus negative & 0.836 & & & NA \\
\hline Size: $\langle 5 \mathrm{~cm}$ versus $\geqq 5 \mathrm{~cm}$ & 0.481 & & & NA \\
\hline Location: cardia versus body versus antrum & 0.338 & & & NA \\
\hline Invasion depth: $\mathrm{T} 1$ versus $\mathrm{T} 2$ versus $\mathrm{T} 3$ versus $\mathrm{T} 4$ & 0.031 & 0.912 & $0.597-1.392$ & 0.668 \\
\hline LNM: N0 versus N1 versus N2 versus N3a versusN3b & $<0.001$ & 1.604 & $1.125-2.286$ & 0.009 \\
\hline Liver metastasis: yes versus no & 0.938 & & & NA \\
\hline Hotair: high versus low & $<0.001$ & 0.47 & $0.222-0.997$ & 0.04 \\
\hline Invasion of contiguous organs: yes versus no & 0.845 & & & NA \\
\hline Microvessel invasion: yes versus no & 0.176 & & & NA \\
\hline Stage: I versus II versus III versus IV & $<0.001$ & 5.009 & $1.469-17.081$ & 0.01 \\
\hline Lauren's classification: diffuse versus intestinal & 0.016 & 1.441 & $0.793-2.618$ & 0.231 \\
\hline Grade of differentiation: Well and moderate versus poor & 0.76 & & & NA \\
\hline Preoperative chemotherapy: yes versus no & 0.009 & 1.567 & $0.85-2.891$ & 0.15 \\
\hline CEA (ug/mL): $\leqq 5$ versus $>5$ & 0.956 & & & NA \\
\hline CA199 (U/mL): $\leqq 37$ versus $>37$ & 0.51 & & & NA \\
\hline
\end{tabular}

Abbreviations: CI, confidence interval; NA, not adapted; LNM, lymphatic nodes metastasis.
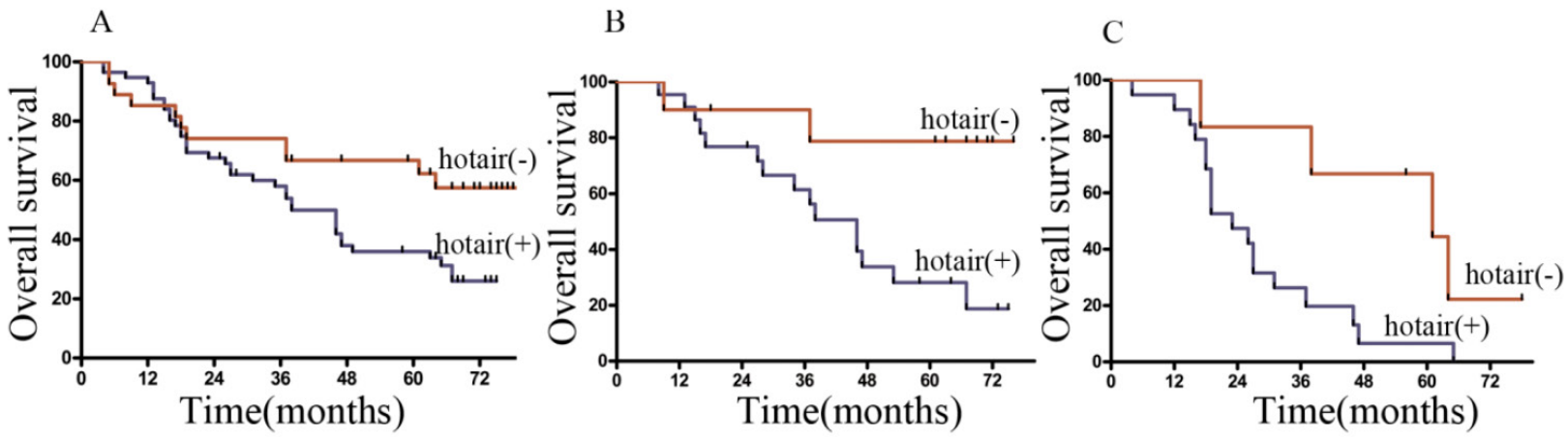

Fig 6. Overall survival curves of GC patients according to the HOTAIR expression levels. (A) Kaplan-Meier survival curves of the whole cohort of 83 patients (B) Kaplan-Meier survival curves of the patients in N2 stage. (C) Kaplan-Meier survival curves of the patients in N3a stage.

\section{Discussion}

Accumulating evidences have revealed that HOTAIR is misregulated in different types of cancer. For example, HOTAIR expression is increased in pancreatic tumors compared with nontumorous tissue and HOTAIR knockdown results in decreased cell proliferation, altered cell cycle progression and induces apoptosis [14]. In like manner, HOTAIR expression is also increased in HCC [13] and human breast cancer. It was demonstrated that if cells expressing HOTAIR were grafted into mouse mammary fat pads, a modest increase in the rate of primary tumor growth was observed [12]. Furthermore, Over-expression of HOTAIR was also strongly associated with high-risk grade among gastrointestinal stromal tumor (GIST) specimens [18].These lines of evidence imply a possible role of HOTAIR as a pro-oncogene in a variety of human cancers. In present study, we examined the expression pattern of HOTAIR in GC patients and investigated its clinical implications. Realtime RT-PCR showed that the HOTAIR was highly up-regulated in GC tumor tissues compared with adjacent nontumorous tissues. Moreover, up-regulated HOTAIR expression was heterogeneous among patients and related to various clinicopathological factors including TNM stage and LNs metastasis. The potential role of HOTAIR in GC growth, in vitro, had also been examined in this study, however, our result showed that altered HOTAIR expression in AGS cells had no significant impact on either viability or apoptosis (data not 
showed). Our observation contrasted sharply with previous studies regarding the role of HOTAIR in regulating cell proliferation. This phenomenon suggested that the function of HOTAIR might have tissue specificity.

HOTAIR is one of the first lncRNAs described to have a fundamental role in cancer metastasis. High levels of HOTAIR expression were found to be correlated with both distal metastasis and lymph node metastasis in HCC [19]. Similarly, our present study found that the high expression of HOTAIR was significantly associated with LNs metastasis. ROC curve showed that the AUC value was nearly 0.8 which indicated that the expression level of HOTAIR might be used to predict the LNs metastasis in patients with GC. Furthermore, to further confirm the role of HOTAIR in GC progression, we employed siRNA technology to suppress the expression of HOTAIR in a GC cell line, AGS, which expresses high level of endogenous HOTAIR. The vitro assays showed that HOTAIR depletion in the GC cells reduced cancer invasiveness significantly.

In addition, our present study tried to identity a "metastasis signature" of genes that support the role of HOTAIR in promoting invasion. As depicted earlier, over-expression of HOTAIR in breast cancer cell lines is accompanied by an altered chromatin state, recruiting the PRC2 to the HOXD locus which leads to transcriptional silencing across $40 \mathrm{~kb}$. So it is possible that changes in HOTAIR may cause aberrant regulation of a broad subset of genes involved in cancer metastasis. Among the candidate genes of "metastasis signature", different members of MMPs family can remodel extracellular matrix and degrade the basement membrane, thereby favoring invasion. In addition, members of the plasminogen system and motility-related genes are also supposed to be involved in multiple steps of invasion-metastasis cascade [20]. So, these selected genes were enrolled for our analysis. By using realtime RT-PCR, we showed that high expression of HOTAIR was positively correlated with up-regulation of ICAM-1 and some members of MMPs family including MMP1, MMP3, and MMP9. Our in vitro study also demonstrated that MMP1 and MMP3 could be suppressed by HOTAIR knockdown, implying their participation in HOTAIR induced metastasis.

The functional importance of HOTAIR for the activation of invasion indicates that further studies should identify the role of this lncRNA in context of EMT [21]. EMT processes, characterized by diminished epithelial characteristics and increased mesenchymal attributes, endow epithelial cells with enhanced migratory potential and have implicated in many physiological and pathological processes re- quiring cell migration [22]. Though lacked direct supporting evidence, the role of HOTAIR in regulating EMT had been suspected. For example, HOTAIR remodels the gene expression pattern of breast epithelial cells to a pattern more resembling embryonic fibroblasts [12] . Furthermore, the expression of snail, one of major transcript factors regulating EMT, can be induced by HOTAIR overexpression [12]. Additionally, another evidence supporting the involvement of HOTAIR in EMT is the interaction between twist and miR-10b. MiR-10b is a microRNA transcribed from the $3^{\prime}$ HOXD locus and can be induced by Twist [23]. However, few previous studies have provided direct evidence to elucidate the actual contribution of HOTAIR to the metastatic cascade in the context of EMT. In this study, we compared EMT-associated genes between HOTAIR (High) and HOTAIR (Low) tissues, our results showed that most of the epithelial markers were down-regulated, whereas, the mesenchymal markers were up-regulated in HOTAIR (High) patients. Our in vitro study also confirmed that deletion of HOTAIR could reverse EMT, implying HOTAIR could act as possible regulators of EMT. Furthermore, we demonstrated that the effect of HOTAIR inhibition on tumor cell invasion could be restored by exogenous snail expression which could elicit a complete EMT process. Taken together, these findings suggested that HOTAIR might promote EMT through regulating snail.

Most importantly, in 83 GC patients treated with D2 radical resection, high expression level of HOTAIR in tumors was associated with poor survival. Multivariate analyses further revealed that HOTAIR expression was an independent prognostic factor for overall survival. In addition, when stratified by lymphatic nodes status, patients with low expression of HOTAIR, both in N2 and N3a stages, had significantly longer mean survival times than those with high expression of HOTAIR. Our findings were consistent with earlier reports which correlated elevated expression of HOTAIR with the poor progression and prognosis of patients. For example, high HOTAIR expression level is an independent prognostic marker for HCC recurrence and shorter survival in the patients who received a liver transplantation [13].

\section{Conclusion}

In summary, our present study showed that high expression of HOTAIR was a strong predictor of both LNs metastasis and postoperative survival in patients with GC. It is likely to be a useful biomarker for diagnostic and/or prognostics. Moreover, the finding that HOTAIR promoted EMT through regulating snail expression could add our understanding of the molecular mechanisms by which HOTAIR contribute 
to tumor invasion. Most importantly, HOTAIR may be used as molecular targets for the discovery of antitumor drugs against human GC metastasis.

\section{Supplementary Material}

Table S1.

http://www.ijbs.com/v09p0587s1.pdf

\section{Acknowledgment}

This study was supported by the Zhejiang provincial natural science foundation [Grant No. LY12H16029, LY13H160027], key project of Provincial Administration of Traditional Chinese Medicine [Grant No. 2012ZZ002], and technological project of Zhejiang province [Grant No. 2012C23114]. We thank the surgeons and nurses who kindly facilitate the recruitment and collection of patient information.

\section{Competing Interests}

The authors have declared that no competing interest exists.

\section{References}

1. Jemal A, Siegel R, Ward E, et al. Cancer statistics, 2008. CA Cancer J Clin. 2008; 58: 71-96.

2. Villanueva MT. Combination therapy: update on gastric cancer in East Asia. Nat Rev Clin Oncol. 2011; 8: 690.

3. Lin Y, Ueda J, Kikuchi S, et al. Comparative epidemiology of gastric cancer between Japan and China. World J Gastroenterol. 2011; 17: 4421-4428.

4. Stein LD. Human genome: end of the beginning. Nature. 2004; 431: 915-916.

5. Brosnan CA, Voinnet O. The long and the short of noncoding RNAs. Curr Opin Cell Biol. 2009; 21: 416-425.

6. Costa FF. Non-coding RNAs: new players in eukaryotic biology. Gene. 2005; 357: 83-94.

7. Calin GA, Croce CM. MicroRNA signatures in human cancers. Nat Rev Cancer. 2006; 6: 857-866.

8. Popov N, Gil J. Epigenetic regulation of the INK4b-ARF-INK4a locus: in sickness and in health. Epigenetics. 2010; 5: 685-690.

9. Calin GA, Liu CG, Ferracin M, et al. Ultraconserved regions encoding ncRNAs are altered in human leukemias and carcinomas. Cancer Cell. 2007; 12: 215-229.

10. Gutschner T, Diederichs S. The Hallmarks of Cancer: A long non-coding RNA point of view. RNA Biol. 2012 Jun;9(6):703-19.

11. Rinn JL, Kertesz M, Wang JK, et al. Functional demarcation of active and silent chromatin domains in human HOX loci by noncoding RNAs. Cell. 2007; 129: 1311-1323.

12. Gupta RA, Shah N, Wang KC, et al. Long non-coding RNA HOTAIR reprograms chromatin state to promote cancer metastasis. Nature. 2010; 464: 1071-1076.

13. Yang Z, Zhou L, Wu LM, et al. Overexpression of long non-coding RNA HOTAIR predicts tumor recurrence in hepatocellular carcinoma patients following liver transplantation. Ann Surg Oncol. 2011; 18: 1243-1250.

14. Kim K, Jutooru I, Chadalapaka G, et al. HOTAIR is a negative prognostic factor and exhibits pro-oncogenic activity in pancreatic cancer. Oncogene. 2012.

15. Ray PS, Wang J, Qu Y, et al. FOXC1 is a potential prognostic biomarker with functional significance in basal-like breast cancer. Cancer Res. 2010; 70: 3870-3876.

16. Xu ZY, Ding SM, Zhou L, et al. FOXC1 contributes to microvascular invasion in primary hepatocellular carcinoma via regulating epithelial-mesenchymal transition. Int J Biol Sci. 2012; 8: 1130-1141.

17. Cheng J, Xie HY, Xu X, et al. NDRG1 as a biomarker for metastasis, recurrence and of poor prognosis in hepatocellular carcinoma. Cancer Lett. 2011; 310: 35-45.
18. Niinuma T, Suzuki H, Nojima M, et al. Upregulation of miR-196a and HOTAIR drive malignant character in gastrointestinal stromal tumors. Cancer Res. 2012; 72: 1126-1136.

19. Geng YJ, Xie SL, Li Q, et al. Large intervening non-coding RNA HOTAIR is associated with hepatocellular carcinoma progression. J Int Med Res. 2011; 39: 2119-2128.

20. Valastyan S, Weinberg RA. Tumor metastasis: molecular insights and evolving paradigms. Cell. 2011; 147: 275-292.

21. Gibb EA, Brown CJ, Lam WL. The functional role of long non-coding RNA in human carcinomas. Mol Cancer. 2011; 10: 38.

22. Bonnomet A, Brysse A, Tachsidis A, et al. Epithelial-to-mesenchymal transitions and circulating tumor cells. J Mammary Gland Biol Neoplasia. 2010; 15: 261-273.

23. Wan Y, Chang HY. HOTAIR: Flight of noncoding RNAs in cancer metastasis. Cell Cycle. 2010; 9: 3391-3392. 